\title{
Adorning Web Search based on Ontology Semantics Spaces Provisioned through Web Services
}

\author{
P. Nandhakumar \\ Research Scholar \\ Karpagam University \\ Coimbatore, Tamil Nadu, India
}

\author{
M. Hemalatha \\ Dept. of Computer Science, \\ Karpagam University \\ Coimbatore, Tamil Nadu, India
}

\author{
Kashyap Dhruve \\ Technical Director \\ Sovereign Technologies
}

\begin{abstract}
The data available on the World Wide Web is assumed to be humongous and infinite. Search Engines have emerged to be integral tools of information retrieval from the web. The current search engines available process queries and produce results based on the location and information occurrence on the web pages providing unsatisfactory results [8]. Web 3.0 and the semantic web incorporated data on the web in machine readable form providing better search results. This paper introduces a semantic search framework named Semantic Search Framework using Semantic Web Services (SSFSWS) built on service oriented architecture (SOA) targeted to enhancing search responses. The framework consists of semantic search providers offering semantic search services. The semantic search services are composed using the depth first search algorithm. The semantic search services offered rely on the RDF data and its corresponding Ontologies built to provide search responses. An OWL2 language namely SROIQ-DL is considered to build the ontologies and represent complex description logic that exists in the RDF data. The framework also introduces effective caching strategies adopted to improve response times. The framework introduced provides ranking schemes based on the ontology relevance scores of the responses observed. A prototype implementation of the SSFSWS is discussed and its benefits over the existing semantic search engine are clearly discussed in this paper.
\end{abstract}

\section{Keywords}

Semantic Web, Semantic Web Search, SOA, Ontologies, OWL 2, Semantic Web Services, Semantic Web Service Composition.

\section{INTRODUCTION}

The explosive growth of the World Wide Web (WWW) [1] has provided extensively large amounts of data available. Search Engines which provide information from the WWW based on the queries have become vital tools. The major shortcoming of the current search engines is that the search results provided are not always true as there exist's no relation derivation from the search queries and the presented search results. The foremost initiative of the semantic web [6] is to broaden the current human understandable data on the www by encoding some of the semantics of resources in a machine understandable form. On achieving representation of the www data in a machine understandable form provides newer avenues of more additional functionalities and advanced application on the web. Semantic Web application would be able to process information, search for information, integrate and present the information of these applications in a meaningful manner. Resource Description Frameworks (RDF) was introduced as the database of the semantic web [2] by the
World Wide Web Consortium (W3C). The Web Ontology Language (OWL) [3] was introduced to represent the relations and the semantics exhibited by the RDF database in a better machine understandable format. The framework proposed in this paper utilizes the $S R O I Q-D L$ [9] an OWL2 [4] ontology description language for better machine understandable knowledge representation. The OWL2 ontology utilized in the proposed framework is also effective in handing logical and conditional search queries as discussed in the future sections of this paper. A desired feature of semantic search is its capability to provide additional informative results when compared to standard search engines. Standard search engines available provide results based on the information available with a web page on the other hand semantic search provide information contained in the web page along with some relational aspect functions which improves the search results provided. An additional feature of the semantic search engine is its ability to provide for refined user preferences for further search criteria based on the current search criteria.

The semantic web is envisioned to facilitate integration of data available across various web applications, web servers through semantic web services [6] [7]. The semantic information available with the service providers is in the RDF format. Additionally the ontologies built based on the RDF data provide shared concepts and relations using either OWL1 or OWL2 ontology description languages. We define such service providers as semantic service providers. To facilitate additional descriptive logics amongst the entities the framework described in this paper considers OWL2 when compared to its predecessor OWL1 which provides limited support. The interoperability of the semantic information available with the web applications is a fundamental requirement which leads to providing semantic search as a service apart from additional semantic web services for applications. Semantic Web Services are similar to conventional web services which provide machine to machine interaction over the www between a web server and a web client. Semantic web services use markup languages to provide data in a machine readable form with all the relational details (ontology details). The SSFSWS introduced in this paper provides a semantic search environment built on service oriented architecture (SOA). The architecture of the SSFSWS is as shown in Figure 1.

The remaining manuscript is organized as follows. Section 2 of the paper discusses the literature review undertaken and the work of fellow researchers. The next section introduces the SSFSWS in which the semantic search providers, the semantic search application and the depth first search algorithm adopted for semantic web service composition is discussed in detail. Section 4 of this paper discusses the experimental study undertaken and the conclusion and future work is discussed in the last section of the paper. 


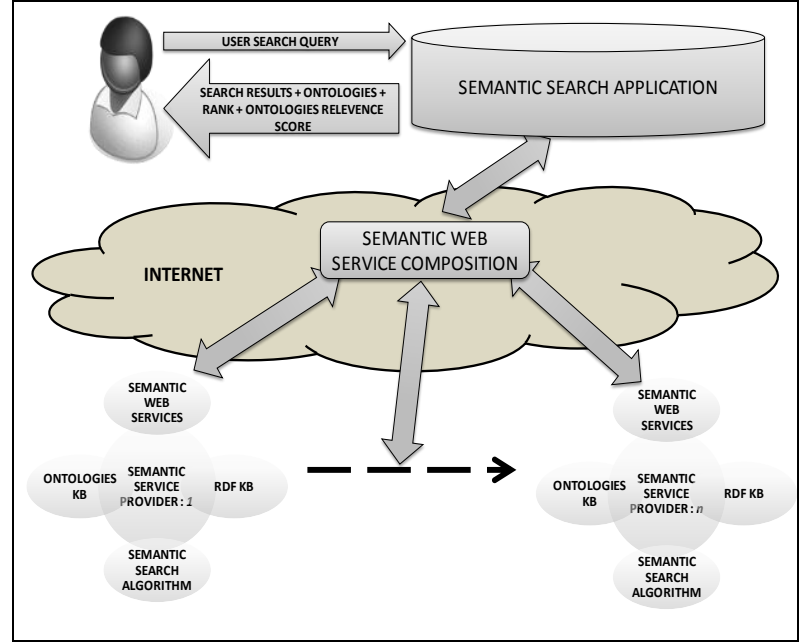

Figure 1: SSFSWS System Architecture

\section{LITERATURE REVIEW}

The semantic web envisions the web content to be in a machine understandable form. Representation the web content in the RDF form has been standardized by W3C [2]. Searching the RDF web content put forth the existence of semantic search engines [21]. Early attempts by researchers [10] to semantically alter the search queries to provide better search responses were introduced. Researchers have proposed search engines based on the RDF knowledge bases [11][12][13]. Ontologies based semantic search engines proved to provide higher accuracy [14] [15] [16]. Hybrid techniques for providing better search results have also been studied during the course of the research work presented here. In hybrid techniques in addition to RDF data Ontologies extracted are also used to provide for better search environments. [17][18][19]. It could be concluded that hybrid techniques perform better than the other classes of semantic search engines hence the SSFSWS utilizes a hybrid technique of providing search responses based on the RDF data and the Ontologies extracted [20]. The framework discussed in this paper considers the semantic search provided through web services [22].

Ranking of the semantic search results have been achieved using various techniques like extended information retrieval techniques [23], interpretations [24], file rank matrices [25] and concept based ranking [26]. Form the study it can be concluded that ontology based ranking mechanism would be idyllic. The framework proposed in this paper considers an Ontology Relevance Score based ranking system. Image Search based on semantics is considered as a hot topic of research currently [27][28]. The future work of the SSFSWS proposed in this paper could be considered to support image based search.

\section{SEMANTIC SEARCH FRAMEWORK using SEMANTIC WEB SERVICES}

This section of the paper discusses the SSFSWS framework proposed. The SOA chosen enable us to realize the framework using a modularized approach. The SOA shown in Fig. 1 could be considered as a complex system of $p: q$ dependencies. Where $p$ represents the services offered and $q$ represents the applications offered by the SOA system. In SOA an application may need multiple service offerings or varied applications need similar services or similar applications may be provided by varied services. The SSFSWS utilizes a similar application of semantic search provided by the $n$ semantic search providers hence it could be said that the SSFSWS depends on the availability of the semantic search application offered by the $n$ semantic search providers. Semantic web service management tends to be cumbersome if it is done manually. In order to automate the semantic web service management we need a common syntax and a common semantic service description to interoperate. The W3C have standardized the syntax definition through the Web Service Description Language (WSDL) [29]. Semantic Interface Description language adopted by the SSFSWS is OWL-S [30]. Let us consider a set of all semantic concepts $S_{C}$ defined

$$
S_{C}=\left\{s_{c_{1}}, s_{c_{2}}, s_{c_{3}}, \ldots \ldots \ldots s_{c_{a}}\right\}
$$

Where $s_{c_{a}}$ represents the $a^{t h}$ semantic concept. The $s_{c_{a}}$ is a concept derived from the RDF $r_{c_{a}} \in R_{C}$ knowledge base (KB) and the ontologies $o_{c_{a}} \in O_{C}$ derived from the RDF knowledge base $R_{C}$. In other words

$$
s_{c_{a}}=f_{c}\left(r_{c_{a}}, o_{c_{a}}\right)
$$

Where $o_{c_{a}}=f_{O}\left(R_{C}, r_{c_{a}}\right) f_{O}$ Represents the ontology building function using SROIQ $(D L)$. The ontology building function extracts all the related concepts and axioms of $r_{c_{a}}$ present in the RDF KB $R_{C}$. The SSFSWS represents a complex SOA hence the RDF $R_{C}$ data set is available with $n^{\text {th }}$ semantic service providers. The RDF data can be defined as $R_{C}=R_{C 1} \cup R_{C 2} \cup R_{C 3} \cup \ldots \ldots . . R_{C n}$

Where $R_{C 1} \neq R_{C 2} \neq R_{C n}$

$$
\begin{aligned}
& R_{C}=\left\{r_{c_{11}}, r_{c_{12}}, \ldots . . r_{c_{1 a}}\right\} \cup\left\{r_{c_{21}}, r_{c_{22}}, \ldots . . r_{c_{2 a}}\right\} \\
& \cup \ldots \ldots . . .\left\{r_{c_{n 1}}, r_{c_{n 2}}, \ldots . . r_{c_{n a}}\right\}
\end{aligned}
$$

The ontologies extracted or the ontology knowledge base could be defined as $O_{C}=O_{C 1} \cup O_{C 2} \cup \ldots . \cup \ldots \ldots O_{C n}$ where $O_{C n}$ the ontology set available with $n^{\text {th }}$ semantic service provider. The locally available ontologies could be defined as $O_{C n} \propto R_{C}$ from the above definition it is clear the ontologies available with semantic service provider $n$ may not contain all the possible concepts, relations and axioms as the complete RDF set $R_{C}$ is unavailable with the $n^{\text {th }}$ semantic service providers. This is the problem that exists in the current semantic search deployments available. The purpose of the SSFSWS is to overcome the short comings by using efficient searching algorithms and semantic web service compositions.

\subsection{Semantic Search Providers}

The semantic search providers in the SSFSWS provide semantic search web services which support the semantic search application. The semantic search providers are defined as $\quad P R=\left\{P r_{1}, P r_{2}, P r_{3}, \ldots \ldots . P r_{n}\right\}$ where $P r_{n}$ is the $n^{\text {th }}$ semantic search providers. The system architecture of the semantic search providers is shown in Figure 2. 


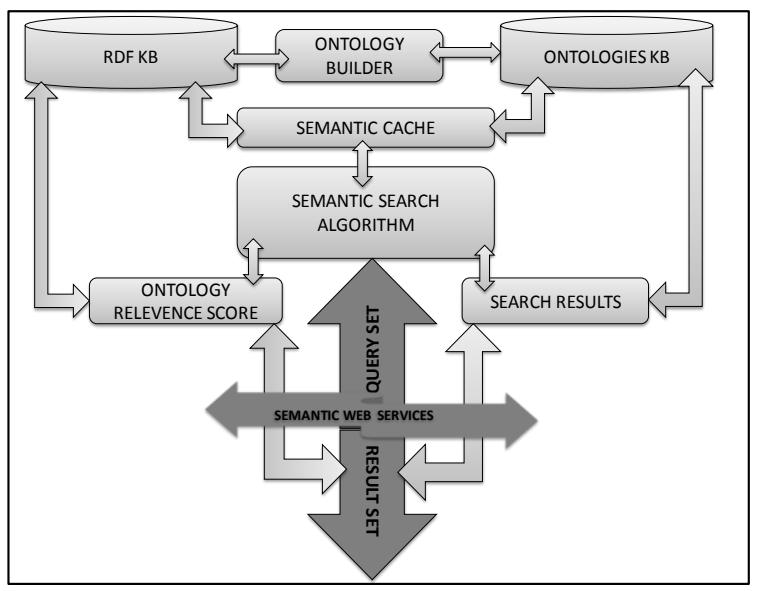

Figure 2: System Architecture of Semantic Search Providers

Each semantic search providers possess the RDF KB. The RDF KB could be represented as

$R_{K B}=\left\{r k b_{1}, r k b_{2}, r k b_{3}, r k b_{4}, \ldots \ldots \ldots . r k b_{r}\right\}$

Where $r k b_{r}$ is the $r^{\text {th }}$ RDF data record available with the semantic search provider $P r_{n} \in P R$.

The RDF data is usually embedded in XML documents as they support segment declaration using tags. The RDF data usually is enclosed within tags represented as $\langle r d f: R D F\rangle$ and $</ r d f: R D F>$ [31]. The RDF records are said to consist consists of triplets [32]. Based on the concept of triplets the record $r k b_{r}$ could be represented as $r k b_{r}=<t r k b_{r_{s u b}}, t r k b_{r_{p r d}}, t r k b_{r_{o b j}}>$ Where $t r k b_{r_{s u b}}$ is the subject triplet, $t r k b_{r_{p r d}}$ is the predicate triple and $t r k b_{r_{o b j}}$ represents the object triplet.

The concepts extracted from the RDF KB include some complex relations that cannot be represented in RDF alone hence the SSFSWS presented here adopts representation of the ontologies through SROIQ Axioms due to its benefits [9] as compared to SHOIN. A brief summary of the syntax and semantics of the SROIQ is as shown in Table 1. The KB of the semantic search providers which constitute of both the RDF KB and Ontology KB are humongous in nature and size. A search executed on huge databases would affect the response times due to numerous disk read and disk write operations involved in the semantic search. To compress the knowledge base and create cache the SSFSWS utilizes a hierarchical data ordering algorithm. The ontology $\mathrm{KB}$ of $r \mathrm{RDF}$ data records is defined as $O_{K B}=\left\{o k b_{1}, o k b_{2}, o k b_{3}, o k b_{4}, \ldots \ldots \ldots o k b_{r}\right\}$

\begin{tabular}{|c|c|c|}
\hline Name & Syntax & Semantics \\
\hline & Concepts & \\
\hline Atomic Concept & $A$ & $A^{I}$ (given) \\
Nominal & $\{a\}$ & $\left\{a^{I}\right\}$ \\
Top Concept & $T$ & $\Delta^{I}$ \\
Negation & $\neg C$ & $\Delta^{I} / C^{I}$ \\
Conjunction & $C \pi D$ & $C^{I} \cap D^{I}$ \\
Existential & $\exists R . C$ & $\left\{x \mid R^{I}\left(x, C^{I}\right) \neq \varnothing\right\}$ \\
Restriction & & \\
Min Cardinality & $\geq n S . C$ & $\left\{x \mid\left\|S^{I}\left(x, C^{I}\right)\right\| \geq n\right\}$ \\
Exists Self & $\exists S$. Self & $\left\{x \mid<x, x>\in S^{I}\right\}$ \\
\hline
\end{tabular}

\begin{tabular}{|lcc|}
\hline \multicolumn{3}{|c|}{ Axioms } \\
\hline Complex Role & $\rho \subseteq R$ & $\rho^{\mathrm{I}} \subseteq R^{\mathrm{I}}$ \\
Inclusion & & \\
$\quad$ Disjoint Roles & $\operatorname{Disj}\left(S_{1}, S_{2}\right.$ & $S_{1}^{I} \cap S_{2}^{I}=\varnothing$ \\
Concept Inclusion & $C \subseteq D$ & $C^{I} \subseteq D^{I}$ \\
Concept Assertion & $C(a)$ & $a^{I} \in C^{I}$ \\
$\quad$ Role Assertion & $R(a, b)$ & $\langle a, b\rangle \in R^{\mathrm{I}}$ \\
\hline
\end{tabular}

\section{Table1: SROIQ Rules Semantic Equivalent}

Let the cache of a concept $s_{c_{a}}$ which represents the $a^{\text {th }}$ semantic concept be represented as Cache $_{s_{c_{a}}}=<s_{c_{a}}$, $r_{c_{a}}, e_{c_{a}}>$

where $r_{c_{a}}$ is the number of relations of the concept and $e_{c_{a}}$ represents the number of edge concepts. It is evident that greater the number of concepts and greater the relations that exist larger is the KB size increasing the number of disk operation for a semantic search. The number of occurrences of a concept in an ontology is directly proportional or equivalent to the number of relations $r_{c_{a}}$ of a concept. Also it can be stated that for a constant $m$ is equivalent to a function of the number of relations $\left(f_{\text {num }} r\right) r_{c_{a}}$ of a concept $s_{c_{a}}$ and a function of the edge depth $\left(f_{\text {edg_dpth }}\right)$ of a concept $s_{c_{a}}$.

$f_{\text {num } \_r}\left(r_{c_{a}}\right) \times f_{\text {edg_dpth }}\left(r_{c_{a}}\right) \approx m$

Also $r_{c_{a}} \approx \sum_{x=1}^{x=m} m / x \approx m \int_{x=1}^{x=m} 1 / x d x=m \ln m$

From the above equation it is clear that even if the number of relations $r_{c_{a}}$ of a concept $s_{c_{a}}$ increase the cache size does not increase by a great extend. Generally the concepts require $2 S_{\text {Unit }}$ storage space per concept $\left(s_{c_{a}}\right)$. The space utilized in storing the cache defined above is given by $\sum_{r_{c_{a}}}(2+$

$\left.f_{\text {num } \_r}\left(r_{c_{a}}\right)\right) \approx S_{U t i l}\left(2+\ln S_{U t i l}\right)$

Where $S_{U t i l}$ is the space required to store the same concept $s_{c_{a}}$. It is considered that only one entry of a $s_{c_{a}}$ concept is allowed in the cache. In order to compare the normal caching strategy with the caching strategy used in SSFSWS the comparison ratio is defined as

$$
\frac{2 S_{U t i l}\left(1+\ln S_{U t i l} / 2\right)}{2 S_{U t i l} \ln S_{U t i l}}=1 / \ln S_{U t i l}+1 / 2
$$

Hence the proposed caching strategy improves the storage space utilization by approximately $50 \%$. The access cost for the caching strategy is defined as

$$
\begin{aligned}
& \text { ACost }_{\text {Cache }}=\sum_{\left\{r_{c_{a}}: f_{\text {num }}\left(r_{c_{a}}\right) \leq t\right\}} f_{\text {num }_{r}}\left(r_{c_{a}}\right) \\
& \approx \int_{S_{U t i l} / t}^{S_{\text {Util }}} \frac{S_{\text {Util }}}{f_{\text {edg_dpth }}} d f_{\text {edg_dpth }}=S_{\text {Util }} \ln t
\end{aligned}
$$

Where $f_{\text {num } \_r}\left(r_{c_{a}}\right) \leq t \leftrightarrow f_{\text {edg_dpth }}\left(r_{c_{a}}\right) \geq S_{\text {Util }} / t$ The probability of AProb finding the concept $s_{c_{a}}$ in the knowledge base is defines as AProb $_{\text {Cache }}=\ln t / \ln S_{\text {Util }}$ The access time of the cache to search for a concept $s_{c_{a}}$ within the knowledge base with a probability $A$ Prob ${ }_{\text {Cache }}$ is defined

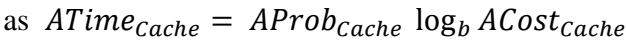

$$
+\left(1-\text { AProb }_{\text {Cache }}\right)\left(\log _{b} \text { ACost }_{\text {Cache }}+1\right)
$$




$$
=\log _{b} \text { ACost }_{\text {Cache }}+\left(1-\text { AProb }_{\text {Cache }}\right)
$$

Where $b$ represents the branching factor of the ontology tree. The cache created based on the RDF KB and Ontology KB is encoded in a binary format for faster access.

The ontology relevance score is a ration between the query concept and the response concept based on the ontologies constructed. The ontology relevance score is used by the Semantic Search Application in ranking the search responses received by the $n$ semantic service provider considered in the SSFSWS. The semantic query $S S_{Q}$ could be defined as a set of concepts and relational operators. The semantic search web service offered supports queries containing Boolean operators like AND ,OR ,NOT ,+, , " "" commonly available with the major search providers. $S s_{Q}=$ $<s_{C_{S S_{Q}}}, R_{c_{S S_{Q}}}>$

The semantic query $s s_{Q}$ could be represented as a $p \times q$ matrix where $p$ represents the number of concepts queried for and $q$ represents the number of relations, logical operators and special characters defined for querying amongst the $p$ concepts. The semantic response $S S_{R}$ is a set of responses and the corresponding ontology relevance score defined as $s s_{R}=<s_{R_{s s_{R}}}, O R S_{R_{s s_{R}}}>$

The semantic response $s s_{R}$ could also be represented as a $r \times r$ matrix where $r$ is the number of responses obtained for the semantic search query $s s_{Q}$. The ontology relevance score is defined as $\operatorname{ors}_{R_{s s_{R}}}\left(s s_{Q}, s s_{R}\right)=\frac{\sum_{r} s_{r_{s s_{R}}}, s s_{Q}}{\left\|s s_{Q}\right\|\left\|s_{r}\right\|}$

To represent the ontology relevance score to a scale of 0 to 1 . Normalization is considered in the SSFSWS hence the ontology relevance score could be defined as

$\operatorname{ors}_{R_{s s_{R}}}\left(s s_{Q}, s s_{R}\right)=s_{r_{s s_{R}}}{ }^{\prime}, s s_{Q}$

Where $s_{r_{s s_{R}}}{ }^{\prime}=\frac{s_{r_{s s_{R}}}}{s_{r}}$

The semantic search providers could be considered as the core of the SSFSWS architecture. The providers discussed in this section not only rely on the RDF KB to provide effective search queries but also rely heavily on the Ontologies KB to provide effective and accurate search responses. The semantic search provider's not only incorporate effective hierarchical caching strategies enhancing query response time but also provide relevant query responses. In addition to the query responses the search providers also provide ontology relevance scores associated with each query responses enabling effective ranking when multiple semantic search responses are composed.

\subsection{Semantic Search Application}

The semantic search application is a user interface which accepts user search queries represented by $S S_{Q}$. The SSFSWS accepts logical, conditional and simple term based search queries. The response of the search is represented as $S S_{R}$. The semantic search application provides the search responses $S S_{R}$ by using semantic web service composition techniques. The depth first search based semantic web service composition algorithm is discussed in the next section of this paper. The semantic search response not only consists of search responses but additionally provides the ontology relevance score used in ranking the search responses i.e. higher the ontology relevance score greater is the rank of the search response. The semantic search application also provides the ontologies constructed after consuming the semantic services provided by the $n$ semantic service providers. The provided ontologies are constructed by the possible concepts and axioms obtained post the semantic web service composition. This enables the SSFSWS to provide better semantic search results and overcome the drawback currently prevalent in the semantic web search sphere (discussed in the previous section of this paper).

Let us consider semantic concept set $S_{C}$ and two concepts $s_{c_{x}} \in S_{C}$ and $s_{c_{y}} \in S_{C}$. There exists 4 possible relations amongst concepts $s_{c_{x}}$ and $s_{c_{y}}$. The possible relations could be defined by using the subsume represented by $S b_{\text {sum }}$ and defined as $S b_{\text {sum }}:\left(S_{C} \times S_{C}\right) \mapsto\{T, F\}$

Where $T$ represents the conditional true relation and $F$ represents a conditionally false relation. Using the above definition we could define the first possible relation between the concepts $s_{c_{x}}$ and $s_{c_{y}}$ as

$S b_{\text {sum }}\left(s_{c_{x}}, s_{c_{y}}\right)=T$ Holds if and only if the semantic concept $s_{c_{x}}$ is a generalization of the semantic concept $s_{c_{y}}$. Also then it could be stated that the semantic concept $s_{c_{y}}$ is a specialization of the semantic concept $s_{c_{x}}$.

$S b_{\text {sum }}\left(s_{c_{y}}, s_{c_{x}}\right)=T$ Holds if and only if the semantic concept $s_{c_{x}}$ is a specialization of the semantic concept $s_{c_{y}}$. Also then it could be stated that the semantic concept $s_{c_{y}}$ is a generalization of the semantic concept $s_{c_{x}}$. If the semantic concepts $s_{c_{x}}$ and the semantic concept $s_{c_{y}}$ are not related then $S b_{\text {sum }}\left(s_{c_{x}}, s_{c_{y}}\right)=F$ and $S_{\text {sum }}\left(s_{c_{y}}\right.$, $\left.s_{c_{x}}\right)=F$. If the semantic concepts $s_{c_{x}}$ and the semantic concept $s_{c_{y}}$ are equal then $S b_{\text {sum }}\left(s_{c_{x}}, s_{c_{y}}\right)=T$ and $\operatorname{Sb}_{\text {sum }}\left(s_{c_{y}}, s_{c_{x}}\right)=T$.

The generalization, specialization and the subsume $S b_{\text {sum }}$ relations are transitive. Let us consider a parameter $p_{x}$ of the semantic service provider $P r_{x}$ and a parameter $p_{y}$ of the semantic service provider $P r_{y}$. If the parameters $p_{x}=$ $p_{y}$ then the semantic web service could be called if only $S b_{\text {sum }}\left(P r_{x}, P r_{y}\right)=T$. It could also be stated that the parameter $p_{x}$ requires less or equal data than the parameter $p_{y}$.For the semantic web service composition required there is no requirement for a demarcation amongst the concepts and the semantic concepts. Let's define a set of semantic web services available with the semantic search application as follows

$$
S w s_{P r}=\left\{s w s_{P r_{1}}, s w s_{P r_{2}}, \ldots \ldots \ldots s w s_{P r_{n}}\right\}
$$

where $s w s_{P r_{n}}$ represents the $n^{\text {th }}$ semantic web service offered by semantic service provider $P r_{n}$.

Each semantic web service offered by semantic service provider $P r_{n}$ required a set of inputs denoted as $s s_{Q_{P r_{n}}}$ and if the set of inputs is provided in an orderly fashion the semantic web service provides a set of output concepts denoted by $s S_{R_{P r_{n}}}$ and $s S_{R_{P r_{n}}} \in S_{C}$. The depth first search semantic 
web service composition algorithm discovers the semantic web services available $S w s_{P r}$. On successful execution of the semantic web service execution algorithm the next semantic web service i.e. $s w S_{P_{2}}$ could be processed only if the execution of the previous $s w s_{P_{1}}$ (provided with the input parameters $S s_{Q_{P r_{1}}}$ and the output concepts $S S_{R_{P r_{1}}}$ are obtained in response) is processed successfully. Let the depth first search based semantic web service composition be represented as $\operatorname{Comp}_{S w s}\left(S w S_{P r}\right)$ then the semantic web service composition is said to successfully process all the requests if

$$
\begin{aligned}
& \operatorname{Comp}_{S w s}\left(S w S_{P r}\right) \leftrightarrow \forall \mathcal{X} \in S s_{Q_{P r_{1}}} \exists \mathcal{Y} \in S S_{Q_{P r}} \\
& : \operatorname{Sb}_{\text {sum }}(x, \mathcal{Y}) \wedge \forall \mathcal{X} \in S s_{Q_{P r_{z}}}, z \in\{2,3, \ldots, n) \\
& \exists \mathcal{Y} \in S S_{Q_{P r}} \cup S S_{R_{P r_{z-1}}} \cup \ldots . . \cup S S_{R_{P r_{1}}} \\
& : S b_{\text {sum }}(\mathcal{X}, \mathcal{Y}) \wedge \forall \mathcal{X} \in S S_{R_{P r_{1}}} \cup \ldots \cup S S_{R_{P r_{n}}} \cup S S_{Q_{P r}} \\
& : \operatorname{Sb}_{\text {sum }}(x, \mathcal{Y})
\end{aligned}
$$

Let $f_{S S W S}$ represent a semantic service provider search function based on a concept $S_{C}$ which provides all the set of semantic web services available defined as $f_{S P r}\left(S_{C}\right)=S w S_{P r}$

Also it could be stated that

$$
\begin{aligned}
\forall s_{c_{a}} \in f_{S P r}\left(S_{C}\right) & \exists S S_{R_{P r_{a}}} \in S S_{R_{P r}} \\
& : S_{\text {sum }}\left(S_{C}, S S_{R_{P r_{a}}}\right)
\end{aligned}
$$

The semantic search application is an interface which provides the search criteria to the composed services the results obtained are then there by provided to the user. On receiving the user's semantic search query $S S_{Q}$ the application of the SSFSWS performs the semantic web services search function $f_{\text {SSWS }}$. The web service offerings amongst the varied semantic service providers are obtained by the advertisement process invoked by the $f_{S S W S}$. Based on the semantic web services offered and the user query appropriate web services are selected. The selected web service offerings $S w s_{P r}$ are composed using the semantic web service composition function $\operatorname{Comp}_{S w s}\left(S w s_{P r}\right)$. On completing the composition the semantic web services are invoked by parsing the required user parameters $S S_{Q}$. The results obtained are aggregated and ranked based on the ontology relevance score. Higher is the ontology relevance score higher is the rank. The ranking could be easily achieved using any sorting algorithm. Let the semantic web search response set be defined as

$S S_{R}=\left\{s S_{R_{P r_{1}}}, s S_{R_{P r_{2}}}, s S_{R_{P r_{3}}}, \ldots . . s S_{R_{P r_{n}}}\right\}$

Where $s s_{R_{P r_{n}}}$ represents the semantic search response received from the $n^{\text {th }}$ semantic service provider for a given query set $S S_{Q}$. As stated earlier the semantic search algorithm available at the semantic service provider's end, provide the result page info, the ontologies behind the search, the ontology relevance score. Based on this argument $S S_{R_{P r_{n}}}$ could be defined as

$s S_{R_{P r_{n}}}=\left\{r_{1} S s_{R_{P r_{n}}}, r_{2} S s_{R_{P r_{n}}}, r_{3} S s_{R_{P r_{n}}} \ldots . r_{m} S s_{R_{P r_{n}}}\right\}$

Where $r_{m} s s_{R_{P r_{n}}}$ represents the $m^{\text {th }}$ search result received from the $n^{\text {th }}$ semantic service provider for a given query set $S S_{Q}$.
The semantic web service composition is an important entity of the semantic web search application. The next section of this paper discusses the depth first search algorithm utilized in composing the semantic web services $S w S_{P r}$ offered by the $n$ semantic service providers.

\subsubsection{Semantic Web Service Composition Using Depth First Search Algorithm}

The semantic search framework SSFSWS introduced in this paper utilizes the depth first search algorithm for semantic web service composition. The dept first search algorithm is selected for the sole purpose of quicker responses it offers and it is computationally lighter when compared to other semantic web service composition algorithms. The web service composition function introduced in the earlier section of this paper $\operatorname{Comp}_{S w s}\left(S w s_{P r}\right)$ receives the set of semantic web services $S w S_{P r}$ over which the composition has to be performed. The semantic web services composition is performed using the depth first search algorithm. Let us define a function $f_{S W S-D S}$ which performs the depth first algorithm is defined as

$f_{S W S-D S}\left(s s_{Q}, s s_{R}, S w s_{t m p}, d_{c}\right)=s w s$

Where $s s_{Q}$ represents the input query set, $s s_{R}$ is the desired response, $S w s_{t m p}$ represents the current temporary semantic web services identified, $d_{c}$ represents the current depth and sws represents the resultant semantic web service identified.

The $f_{S W S-D S}$ is solved by the following algorithm

Step 01: START

Step 02: For Each $\operatorname{Var}_{1} \in s s_{R}$

Step 03: For Each $s w s_{P r} \in f_{S P r}\left(S_{C}\right)=S w s_{P r}$

Step 04: Initialization $s s_{R_{\text {tmp }}}=s s_{R}$

Step 05: $\quad$ For Each $\operatorname{Var}_{2} \in S s_{R_{t m p}}$

Step 06: IF $\exists \operatorname{Var}_{3} \in S s_{R_{P r}}: S b_{\text {sum }}\left(\operatorname{Var}_{2}, \operatorname{Var}_{3}\right)$

Step 07:

Step 08:

Step 09:

Step 10: $s s_{R_{t m p}}=s s_{R_{t m p}} / \operatorname{Var}_{2}$

For Each $\operatorname{Var}_{4} \in S s_{Q_{t m p}}$

Step 11: IF $\exists \operatorname{Var}_{5} \in S s_{Q_{P r}}: S b_{\text {sum }}\left(\operatorname{Var}_{4}, \operatorname{Var}_{5}\right)$

Step 12: $\quad s s_{R_{t m p}}=s s_{R_{\text {tmp }}} \cup \operatorname{Var}_{4}$

Step 13:

Step 14:

Each

Step 15: $\quad s w s_{t m p}=s w s_{P r} \oplus S w s_{t m p}$

Step 16:

Step 17:

Step 18:

Step 19:

Step 20:

Step 21:

IF $s s_{R_{\text {tmp }}}=\{\}$

End IF

Return $s w s_{t m p}$

ELSE

$f_{S W S-D S}\left(s s_{Q}, s s_{R_{t m p}}, s w s_{t m p}, d_{c}+1\right)$

Step 22:

Step 23:

Step 24:

Step 25:

Step 26:

Step 27: End For Each

Step 28: End For Each

Step 29: Return \{\}

Step 30: END

Where $V a r_{1}, \operatorname{Var}_{2}, \operatorname{Var}_{3}, \operatorname{Var}_{4}, \operatorname{Var}_{5}$ represent temporary processing variables and $d_{\max }$ represents the maximum depth. The semantic web service composition function denoted by $\operatorname{Comp}_{S w S}\left(S w S_{P r}\right)$ is realized using the following algorithm Step 01: START 
Step 02: Initialization $d_{\max }=2$

Step 03: DO

Step 04: $S w s_{t m p}=f_{S W S-D S}\left(s s_{Q_{P r_{n}}}, s s_{R_{P r_{n}}},\{\}, 1\right)$

Step 05: $\quad d_{\text {max }}=d_{\text {max }}+1$

Step 06: While $S w s_{\text {tmp }} \neq\{\}$

Step 07: END

The Comp Sws $_{\text {S }}$ composes the semantic web services from the back. In each iteration of the DO-WHILE loop the algorithm checks if a service within the $S w s_{P r}$ could provide the service requested for. In the proposed framework SSFSWS we use the composition algorithm to identify the semantic service providers offering the semantic search web services to support the semantic search application.

The SOA architecture considered for the SSFSWS is described in this section of the paper. The SSFSWS is designed to provide appropriate search responses. The SSFSWS relies on the RDF KB and the Ontologies KB housed as the $\mathrm{KB}$ component of the semantic web service providers for provisioning of the search responses. The semantic search web services offered by the service providers are composed using the depth first search algorithm. The next section of the paper discusses the prototype implementation adopted to realize the SSFSWS.

\section{CASE STUDY AND EXPERIMENTAL EVALUATION}

This section of the paper discusses a small case study undertaken to prove the functional feasibility of the SSFSWS. The case study considered a popular semantic corpus Edubase2 [33][34][35]. The Edubase consists of the records of the educational establishments in England and Whales. This corpus is maintained by the Education Department of the United Kingdom Government [36]. The Edubase2 corpus is huge and in order to construct an SOA architecture the Edubase2 data was split into three categories namely establishments offering primary education, establishments offering secondary education and educational establishments offering higher education and collegiate education. Each corpus resulting from the classification discussed is considered as the RDF KB of the semantic search providers. The SOA adopted to demonstrate the functionality of the SSFSWS is as shown in Figure 3.

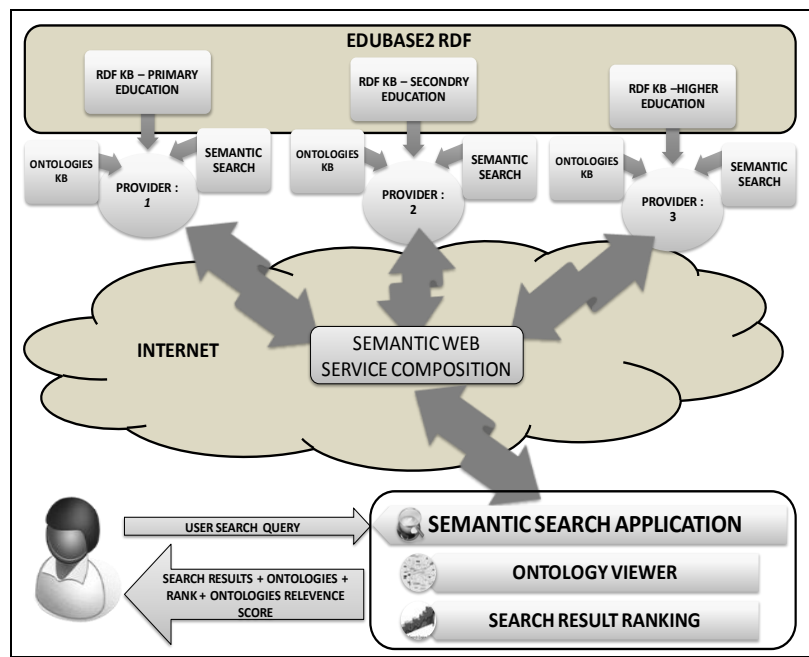

Figure 3: Case Study Architecture Based on EDUBASE
The Microsoft .Net platform 4.0 is considered for the development of the case study presented here. The three semantic service providers offering the semantic search web services were developed on the ASP.Net platform. The web services offered were composed using the depth first search algorithm. The semantic search application was developed on a Windows Presentation Platform using C\#.net and Extensible Application Markup Language (XAML) as the implementation language. The ontology viewer which basically is an interface application to display the ontology graphs resulting from the search query was also developed using C\#.net and XML. Most of the research work done in the area of semantic search preferred a Java based platform for implementation this is a major motivation to develop the SSFSWS on the .Net Platform.

The RDF KB obtained from the Edubase 2 corpus consists of a total number of 66655 records of various educational establishments in England and Whales. A comprehensive data of nearly 218 concepts per school were provided. The corpus on splitting in the terms of the type of education levels provided consists of 24167 establishments offering primary education , 5099 providing secondary education and 37389 establishments offering higher education. The RDF KB housed in the three semantic search providers was considered to build ontologies and represent the relations using SROIQ $D L$. The number of relations extracted based on the RDF KB housed with each semantic search service providers is as shown in Table 2 The number of RDF Records available with each of the semantic search providers is shown in the Figure 4. The average number of relations extracted was found to be around 121. Figure 5 shows the number of relations extracted based on the RDF KB available with the semantic service providers.

\begin{tabular}{|c|l|l|}
\hline Education Type Offered & $\begin{array}{c}\text { Number of } \\
\text { RDF } \\
\text { Records in } \\
\text { Corpus }\end{array}$ & $\begin{array}{c}\text { Number } \\
\text { of } \\
\text { Relations } \\
\text { Extracted }\end{array}$ \\
\hline HIGHER EDUCATION & 37389 & 3663441 \\
\hline SECONDARY EDUCATION & 5099 & 764476 \\
\hline PRIMARY EDUCATION & 24167 & 3653042 \\
\hline
\end{tabular}

Table 2: RDF KB and Ontologies KB Statistics

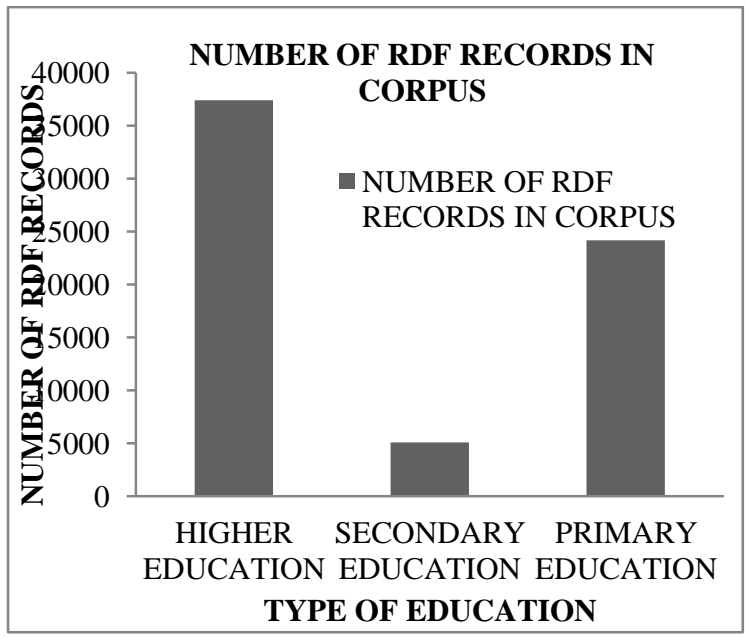

Figure 4: Size of the RDF KB Available with the Semantic Search Providers 
The semantic search application supports ontologies built using the $S R O I Q-D L$ language. A sample ontology obtained after performing the search is shown in Figure 6. The presented case study is compared with the existing semantic search offered using Edubase/Edubase2 corpus maintained by the Education Department of the UK Government. [37][38]. The existing semantic search available online provides no support for relational queries and supports no logical or Boolean operators in the search query. The SSFSWS case study developed over comes these drawbacks and supports logical queries, Boolean operator based queries and queries using special characters. In addition the proposed SSFSWS also provides the ontology view of the response obtained for a query. Ranking the search results based on the ontology relevance score provides more legitimate search responses to be provided to the users.

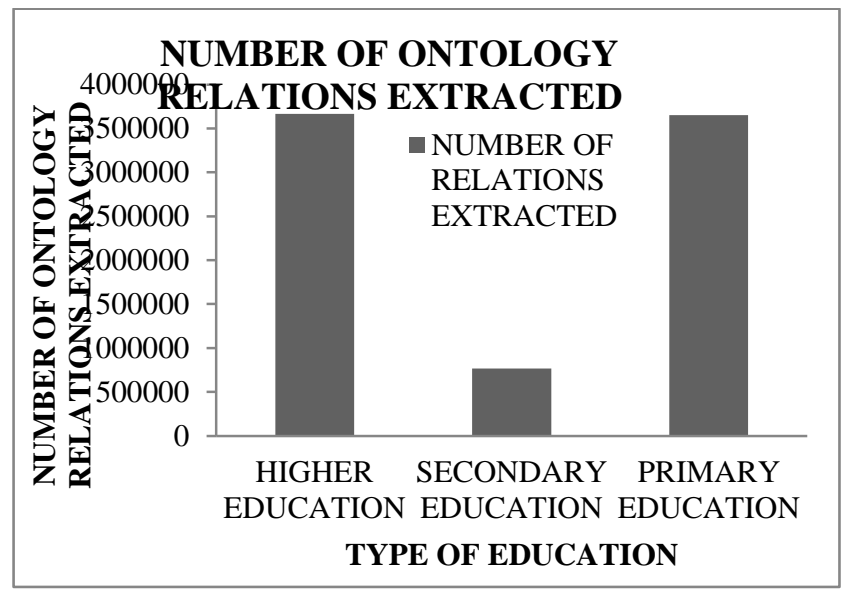

Figure 1: Number of Ontology Relations Extracted to form the Ontology KB

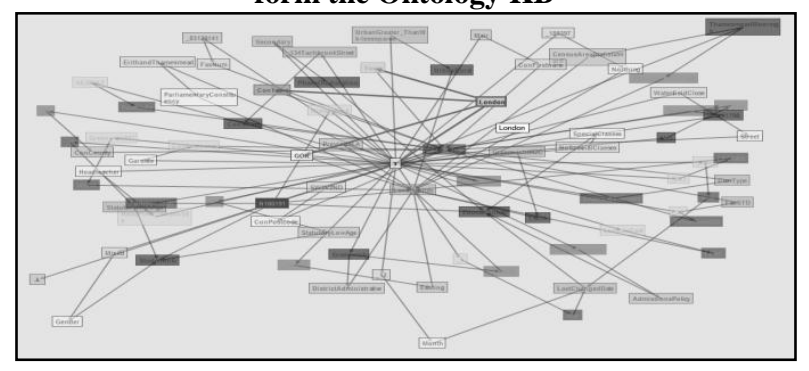

Figure 6: Ontology View Generated at the Semantic Search Application

\section{CONCLUSION AND FUTURE WORK}

This paper introduces a SOA based semantic search framework named SSFSWS. The SSFSWS constitutes of semantic search providers offering semantic search web services and a semantic search application. The semantic search web services are composed using the depth first search algorithm. The semantic search responses in the SSFSWS are based on the RDF KB and the Ontologies KB constructed using the $S R O I Q-D L$ web ontology language. The search response times are optimized by adopting hierarchical caching mechanisms. In addition to optimizing the response times the SSFSWS also provides support for ranking based on the ontology relevance score. The ontologies constructed post the semantic web service composition are also provided as a semantic search response enabling users better graphical representation and analysis for the search query provided. A prototype implementation of the SSFSWS is also discussed and its advantages over the existing semantic search solutions based on the EDUBASE2 are clearly put forth. The future of the SSFSWS could be considered in providing support for image based search and also improving the semantic web service composition algorithm.

\section{REFERENCES}

[1] T. Berners-Lee and M. Fischetti, "Weaving the Web," Chapter Machines and the Web, pp. 177-198, 1999.

[2] W3C 1999 specification."Resource Description Framework (RDF) Model and Syntax Specification",W3C Recommendation 22 February 1999

[3] Web Ontology Language, http://www.w3.org/2004/OWL/

[4] OWL 2 Web Ontology Language, http://www.w3.org/TR/owl2-overview/

[5] T. Berners-Lee, J. Hendler, and O. Lassila, "The SemanticWeb, " Scientific American Magazine, vol. 284, no. 5 , 2001, pp. 34-43

[6] Sheila A. McIlraith, Tran Cao Son, and Honglei Zeng."Semantic Web Services",IEEE INTELLIGENT SYSTEMS,MARCH/APRIL 2001 pp 46-53

[7] M. Burstein, C. Bussler, M. Zaremba, T. Finin, M. N. Huhns, M. Paolucci, A. P. Sheth, and S. Williams, "A Semantic Web Services Architecture" IEEE Internet Comput., vol. 9, no. 5, 2005, pp. 72-81.

[8] Yufei Li, Yuan Wang, and Xiaotao Huang,"A RelationBased Search Engine in Semantic Web",IEEE TRANSACTIONS ON KNOWLEDGE AND DATA ENGINEERING, VOL. 19, NO. 2, FEBRUARY 2007. pp 273-282

[9] Horrocks I, Kutz O, Sattler U. The even more irresistible SROIQ. 2006

[10] Moldovan, D.I. and Mihalcea, R,"Using wordnet and lexical operators to improve internet searches". IEEE Internet Computing 4 (2000) pp 34-43

[11] D. Tümer, M. A. Shah, and Y. Bitirim, An Empirical Evaluation on Semantic Search Performance of Keyword-Based and Semantic Search Engines: Google, Yahoo, Msn and Hakia, 2009 4th International Conference on Internet Monitoring and Protection 2009.

[12] Manola, F. and McBride, B. (2004) 'RDF primer, W3C recommendation 10 February 2004', Technical Report,W3C, http://www.w3.org/TR/rdf-primer/ (200407-15).

[13] Davies, J. and Weeks, R 'QuizRDF: search technology for the semantic web', 37th Hawaii International Conference on System Sciences (HICSS-37 2004), CDROM/AbstractsProceedings, 5-8 January, Big Island, HI, USA, IEEE Computer Society, ISBN 0-7695-2056-1.

[14] SEWISE: $\quad$ www.georges.gardarin.free.fr/Articles /Sewise_NLDB2003.pdf

[15] Heflin, J. and Hendler, J. 'Searching the web with SHOE',Artificial Intelligence for Web Search. Papers from the AAAI Workshop, WS-2000-01,pp.35-40

[16] A. Maedche, B. Motik, L. Stojanovic, R. Studer, and R. Volz, "An infrastructure for searching, reusing and 
evolving distributed ontologies," in Proceedings of WWW '03Budapest, 2003, pp. 439-448

[17] Rocha, C., Schwabe, D. and de Aragao, M.P. 'A hybrid approach for searching in the semantic web', WWW '04: Proceedings of the Thirteenth International Conference on World Wide Web, pp.374-383

[18] Burton-Jones, A., Storey, V.C., Sugumaran, V. and Purao, S. 'A heuristic-based methodology for semantic augmentation of user queries on the web', Conceptual Modeling - ER 2003, 22nd International Conference on Conceptual Modeling, October 13-16, Proceedings, pp.476-489

[19] Amaral, C., Laurent, D., Martins, A., Mendes, A. and Pinto, C "Design and implementation of a semantic search engine for Portuguese", Proceedings of 4th International Conference on Language Resources and Evaluation, LREC 2004, Vol. I, pp.247-250

[20] Christoph Mangold. "A survey and classification of semantic search approaches",Int. J. Metadata, Semantics and Ontology, Vol. 2, No. 1, 2007. pp 23-34

[21] Eetu Makel. "Survey of Semantic Search Research",www.seco.tkk.fi/publications/2005/makelasemantic-search-2005.pdf

[22] F. F. Ramos, H. Unger, V. Larios (Eds.): LNCS 3061, pp. 145-157, Springer-Verlag Berlin Heidelberg 2004

[23] Cohen, S. Mamou, J. Kanza, Y. Sagiv, Y "XSEarch: A Semantic Search Engine for XML" proceedings of the international conference on very large databases, pp 4556, 2003.

[24] E. Kandogan, R. Krishnamurthy, S. Raghavan, S. Vaithyanathan, and H. Zhu, "Avatar semantic search: a database approach to information retrieval," in Proceedings of SIGMOD '06 Chicago, 2006, pp. 790-792

[25] D. Bhagwat and N. Polyzotis, "Searching a file system using inferred semantic links," in Proceedings of HYPERTEXT '05 Salzburg, 2005, pp. 85-87

[26] Yuzhong Qu and Gong Cheng, "Falcons Concept Search: A Practical Search Engine for Web Ontologies",IEEE TRANSACTIONS ON SYSTEMS, MAN, AND CYBERNETICS-PART A: SYSTEMS AND HUMANS, VOL. 41, NO. 4, JULY 2011,pp 810-816

[27] Xiaoou Tang, Ke Liu, Jingyu Cui, Fang Wen, and Xiaogang Wang."IntentSearch: Capturing User Intention for One-Click Internet Image Search", IEEE TRANSACTIONS ON PATTERN ANALYSIS AND MACHINE INTELLIGENCE, VOL. 34, NO. 7, JULY 2012,pp 1342-1353

[28] Xinmei Tian, Yijuan Lu, and Linjun Yang," Query Difficulty Prediction for Web Image Search", IEEE TRANSACTIONS ON MULTIMEDIA, VOL. 14, NO. 4, AUGUST 2012, pp951-962

[29] David Booth and Canyang Kevin Liu. Web Services Description Language (WSDL) Version 2.0 Part 0: Primer. World Wide Web Consortium (W3C), June 26, 2007. W3C Recommendation. Online http://www.w3.org/TR/2007/REC-wsdl20-primer20070626

[30] David Martin, Mark Burstein,Grit Denker,Daniel Elenius,Joseph Giampapa,Drew McDermott,Deborah
McGuinness,Sheila McIlraith,Massimo Paolucci ,Bijan Parsia ,Terry Payne ,Evren Sirin,Naveen Srinivasan and Katia Sycara. "OWL-S 1.2 Release", OWL-based Web Service Ontology.Web-Ontology Working Group at the World Wide Web Consortium, 2008-12.Online : http://www.ai.sri.com/daml/services/owl-s/1.2/

[31] RDF/XML Syntax Specification ,http://www.w3.org /TR/rdf-syntax-grammar.

[32] $\mathrm{N}$

Triples,http://www.w3.org/2001/sw/RDFCore/ntriples.

[33] Edubase RDF, http://source.data.gov.uk/data/education /edubase/2009-08-14/edubase-rdf-r2rc3.tgz

[34] R.Guha, R.McCool and E.Miller "Semantic Search",WWW2003, May 20-24, 2003, Budapest, Hungary.ACM 1.58113-680-3/03/0005 pp 700-709

[35] Jonathan A. Abourbih, Luke Blaney, Alan Bundy, and Fiona McNeill, "A Single-Significant-Digit Calculus for Semi-Automated Guesstimation"Springer-Verlag Berlin Heidelberg ,IJCAR 2010, LNAI 6173, pp. 354-368, 2010

[36] Department of Education, www.education.gov.uk

[37] Department of Education,http://services.data.gov.uk /education/search Accessed May 26th 2012

[38] Department of Education, http://www.education.gov.uk/schools/performance/index. html Accessed September 1st 2012

\section{AUTHORS PROFILE}

P. Nandhakumar completed M.C.A., M. Phil. and currently pursuing Ph.D in computer science at Karpagam University, Coimbatore under the guidance of Dr.M.Hemalatha, Professor and Head, Dept. of Software System, Karpagam University, Coimbatore. He is working as Senior Software Engineer in Easy Design Systems Private Limited, Coimbatore. Presented one paper in International Conference. Area of research is Web Technology and Software Engineering.

Dr. M. Hemalatha completed M.Sc., M.C.A., M. Phil., Ph.D (Ph.D, Mother Terasa women's University, Kodaikanal). She is Professor \& Head and guiding Ph.D Scholars in Department of Computer Science at Karpagam University, Coimbatore. Twelve years of experience in teaching and published more than hundred papers in International Journals and also presented more than eighty papers in various national and international conferences. She received best researcher award in the year 2012 from Karpagam University. Her research areas include Data Mining, Image Processing, Computer Networks, Cloud Computing, Software Engineering, Bioinformatics and Neural Network. She is a reviewer in several National and International Journals.

Mr.Kashyap Dhruve completed Bachelor of Engineering Degree in Electronics and Communication Engineering from Visvesvaraya Technological University Belgaum. $\mathrm{He}$ is currently working as a technical director in Sovereign Technologies. His areas of research interests are Information Security, Image Processing, Analog Design of Sensor Interface Circuits, Data Compression, Wireless Networks, Wireless Sensor Networks, Cognitive Networks. 